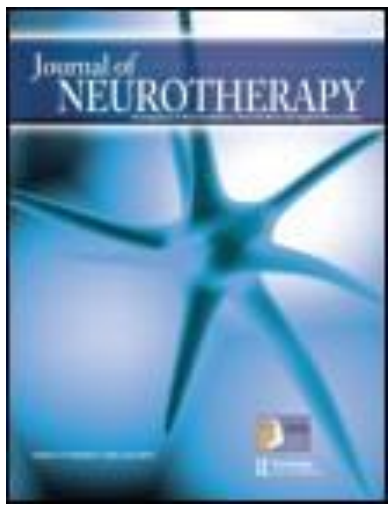

Journal of Neurotherapy:

Investigations in Neuromodulation,

Neurofeedback and Applied

Neuroscience

\title{
The Value of Observational Studies in Neurotherapy
}

Published online: 20 Oct 2008.

To cite this article: (2000) The Value of Observational Studies in Neurotherapy, Journal of Neurotherapy: Investigations in Neuromodulation, Neurofeedback and Applied Neuroscience, 4:3, 1-4, DOI: 10.1300/J184v04n03_01

To link to this article: http://dx.doi.org/10.1300/J184v04n03_01

\section{PLEASE SCROLL DOWN FOR ARTICLE}

(c) International Society for Neurofeedback and Research (ISNR), all rights reserved. This article (the "Article") may be accessed online from ISNR at no charge. The Article may be viewed online, stored in electronic or physical form, or archived for research, teaching, and private study purposes. The Article may be archived in public libraries or university libraries at the direction of said public library or university library. Any other reproduction of the Article for redistribution, sale, resale, loan, sublicensing, systematic supply, or other distribution, including both physical and electronic reproduction for such purposes, is expressly forbidden. Preparing or reproducing derivative works of this article is expressly forbidden. ISNR makes no representation or warranty as to the accuracy or completeness of any content in the Article. From 1995 to 2013 the Journal of Neurotherapy was the official publication of ISNR (www. Isnr.org); on April 27, 2016 ISNR acquired the journal from Taylor \& Francis Group, LLC. In 2014, ISNR established its official open-access journal NeuroRegulation (ISSN: 2373-0587; Www. neuroregulation.org).

THIS OPEN-ACCESS CONTENT MADE POSSIBLE BY THESE GENEROUS SPONSORS

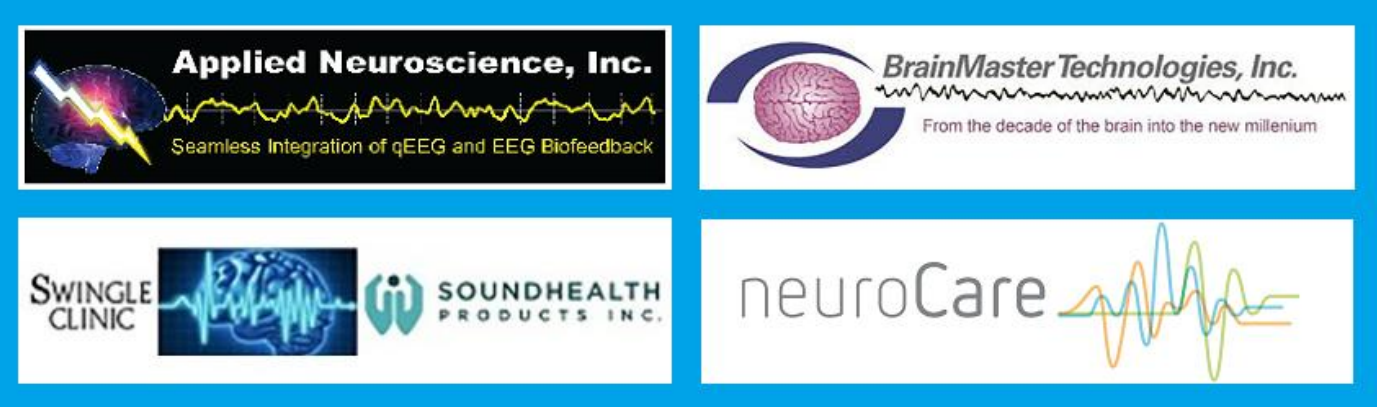




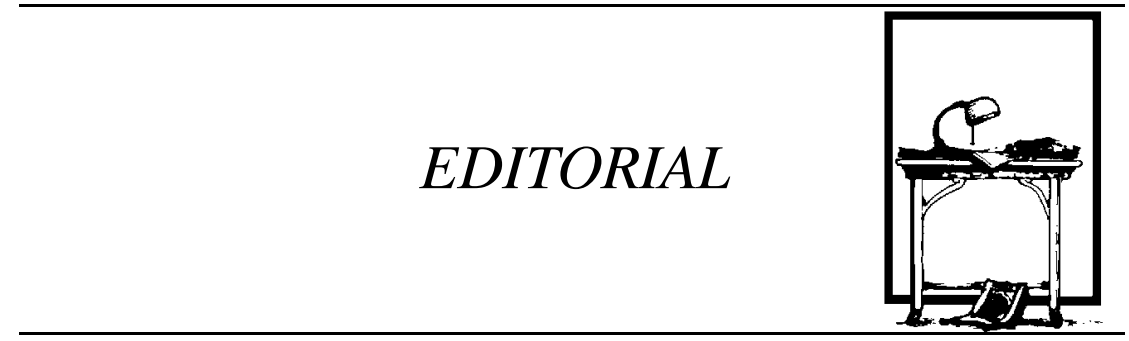

\section{The Value of Observational Studies in Neurotherapy}

One of the perceived shortcomings of neurotherapy is the general lack of randomized prospective studies of brain wave biofeedback, audiovisual stimulation and other neurotherapy modalities. This is due in large part to the difficulty of implementing random assignment to blind study conditions in a clinical practice situation. Most neurotherapy studies in this and other journals are observational, submitted by authors who are clinicians working with participants in practice settings, and in some cases, in academic settings that are also mainly clinical. The managed care revolution in health care makes it increasingly difficult to do clinical research in a practice setting, and those who do so volunteer their time and effort to collect data pertaining to clinical efficacy of treatment methods used in neurotherapy. Only recently has funding become available for studies of "alternative" therapies (through the Institute of Alternative Medicine of the National Institutes of Health) and to date few studies of neurotherapy have been funded. Neurotherapy studies of Attention Deficit Disorder have been held to the same standard as drug studies, but have lacked the randomized clinical trial study design of drug studies. The pharmaceutical industry has supported randomized drug studies 
of ADHD, and these studies have been embraced by academics as definitive, because they leave no room for selection bias. On the other hand, clinically oriented neurotherapists have reported on small observational studies. These studies generally have been underreported in the mainstream literature. Furthermore, these clinical studies have been retrospective reports of treatments not assigned for experimental purposes.

For many years randomized controlled trials have been the only accepted gold standard of efficacy. They are believed to provide much stronger evidence than observational studies such as cohort or case control studies. Two recently published reports in the New England Journal of Medicine (Benson \& Hartz, 2000 and Concato, Shah \& Horwitz, 2000) find that the results of observational studies are very similar to those of controlled clinical trials. This is important news for neurotherapy researchers, who are often unable to undertake expensive and difficult to perform randomized controlled studies.

Benson and Hartz (2000) examined 136 reports of 19 diverse medical and surgical treatments. The studies were contemporary, occurring between 1985 and 1998. In only 2 of the 19 analyses of treatment effects did the combined magnitude of the effect lie outside the $95 \%$ confidence level when observational studies were compared to randomized studies. These authors used four selection criteria for observational studies. The first is that the study was not experimental-that is to say treatments were not assigned for purposes of research, and as such could be retrospective. Secondly, the study looked at differences between two treatments or between one treatment and no treatment. Thirdly, the treatments were prescribed by the health care giver, and not self-prescribed. The fourth is that each of the observational studies included a control group, similar in social demographics and symptom intensity to the treatment group. In most cases the observational studies were done first, with randomized controlled trials following. The general effect of randomized controlled trials was that they confirmed observational studies. Although the authors acknowledge that the fundamental criticism of observational studies is that unrecognized confounding factors may distort the results. "According to conventional wisdom this distortion is sufficiently common and unpredictable that observational studies are not reliable and should not be funded. Our results suggest that observational studies usually do provide valid information."

In a second study, Concato, Shah, and Horowitz (2000) examined metaanalyses of either randomized, controlled trials or those of cohort or case control studies assessing the same intervention. Using this somewhat different approach, they reached a similar conclusion as Benson and Hartz, namely that well designed observational studies are similar in validity to randomized controlled clinical trials. In their words, “the 'average' results from well-designed observational studies (with a cohort or case control design) did not systemically overestimate the magnitude of the associations between exposure and outcome as compared with the results of randomized, controlled 
trials of the some topic." In fact the authors assert that trustworthy results obtained from several quality observational studies may sometimes give the right answers when the results from a single randomized study do not. They suggest that the time-honored hierarchy of research designs (i.e., randomized controlled studies are evidence of the very highest grade and much superior to observational studies) needs to be re-evaluated.

In an accompanying editorial, Pocock and Elbourne (2000) present a counter point to these studies. The stated main concern of this editorial is "if these claims lead to more observational studies of therapeutic interventions and fewer randomized, controlled trials, we see considerable dangers to clinical research and even to the well-being of patients." They search for explanations as to how the two studies of observational design reach conclusions that defy conventional wisdom. After critiquing some aspects of the studies for bias, they conclude, "observational data bases can be useful adjuncts to randomized, controlled trials, to see whether efficacy under controlled conditions in specialist centers translates into effective treatment in routine practice." They assert "although observational studies often are cheaper, quicker, and less difficult to carry out, we should not lose sight of one simple fact: ignorance calls for ... high-quality randomized, controlled trials, not observations that reflect personal choices and beliefs." However, none of the arguments presented that the potential bias of selection in observational studies uniformly invalidates them are compelling. The editorial points out potential weaknesses in the two studies (Benson \& Hartz, 2000 and Concato, Shah \& Horwitz, 2000), but falls short of presenting any solid refutation.

What does this empirical support of observational studies mean for neurotherapy research and reporting? For one thing, there is much data in clinical practice databases that can be examined for consistency and application to a case cohort observational study. This applies to studies of ADHD, learning disabilities, addictive disorders, post-concussive disorder, optimization of performance, anxiety, affective disorders and other clinical applications of neurotherapy. Clinicians, students and academics interested in neurotherapy may have access to databases that can be developed into more important and valid studies of treatment efficacy. These retrospective case cohort studies, if well designed, can stand on their own merits. They will add to the growing body of valid studies showing efficacy. They could be the basis for fundability of longer-term large randomized multi-center controlled studies.

Most importantly, the evidence that observational studies can be as valid as randomized studies supports the credibility of published reports of neurotherapy efficacy that are based on observational design. This should give pause for reflection to care providers and care managers as they consider treatment options of demonstrated efficacy for individual treatment plans.

David L. Trudeau, MD 


\section{REFERENCES}

Concato, J., Shah, N. \& Horowitz, R. (2000). Randomized controlled trials, observational studies, and the hierarchy of research designs. New England Journal of Medicine, 342(25), 1887-1892.

Benson, K., \& Hartz, A.J. (2000). A comparison of observational studies and randomized controlled trials. New England Journal of Medicine, 342(25), 1878-1886.

Pocock, S.J. \& Elbourne, D.R. (2000). Randomized trials or observational tribulations? (Editorial) New England Journal of Medicine, 342(25), 1907. 